\title{
On a Simple Type of Integro-differential Equation
}

\author{
By R. P. Gillespie, Glasgow University. \\ (Received 28th July, 1934. Read 2nd November, 1934.)
}

In a previous paper ${ }^{1}$ in these Proceedings the author discussed conditions for a maximum or minimum of functions of integrals of the type

$$
\phi\left[\int_{x_{1}}^{x_{2}} f\left(x, y, y^{\prime}\right) d x, \int_{x_{1}}^{x_{2}} g\left(x, y, y^{\prime}\right) d x\right],
$$

using the methods of the Calculus of Variations. In the effort to establish a third necessary condition for a minimum-the analogue of Jacobi's condition in the ordinary variational problem ${ }^{2}$-it was found that the analogue of Jacobi's Equation was an integrodifferential equation of the form

$\frac{d^{2} u}{d x^{2}}+l(x) \frac{d u}{d x}+m(x) u+n(x) \int_{x_{1}}^{x_{2}}\left[p(x) u+q(x) \frac{d u}{d x}\right] d x=\mathbf{0}$.

In the ordinary variational problem Jacobi's Equation is a second order homogeneous linear differential equation, and the important property is Sturm's result that the zeros of two linearly independent solutions are interlaced. In the present paper a method of solving equation (1) will be given, and it will be shown that any solution of the equation can be expressed in terms of linearly independent solutions, whose zeros are interlaced exactly as in the case of the ordinary differential equation. This is the property which was required for the variational problem.

$\S 1$. It is assumed that the functions $l(x), m(x), n(x), p(x), q(x)$ in equation ( 1 ) are continuous in the range with which we are dealing. Now if $u=u(x)$ is any solution of equation (1), the expression

$$
\int_{x_{1}}^{x_{2}}\left[p(x) u+q(x) \frac{d u}{d x}\right] d x
$$

1 R. P. Gillespie, Proc. Edinburgh Math. Soc. (2), 3 (1932), 87-98.

2 Cf. O. Bolza, Vorlesungen über Variationsrechnung (1909), 68-87. 
where $u=u(x)$, has a definite value, $\lambda$ say, independent of $x$. Thus $u=u(x)$ satisfies the differential equation

$$
\frac{d^{2} u}{d x^{2}}+l(x) \frac{d u}{d x}+m(x) u+\lambda n(x)=0,
$$

so that each solution of equation (1) is also a solution of a certain second order differential equation. If

$$
a u_{1}(x)+\beta u_{2}(x)
$$

is the general integral of the equation

$$
\frac{d^{2} u}{d x^{2}}+l(x) \frac{d u}{d x}+m(x) u=0,
$$

where $u_{1}(x)$ and $u_{2}(x)$ are linearly independent integrals and $a$ and $\beta$ are arbitrary constants; and if $u_{3}(x)$ is any particular integral of the equation

$$
\frac{d^{2} u}{d x^{2}}+l(x) \frac{d u}{d x}+m(x) u+n(x)=0,
$$

then it is clear that

$$
u=\alpha u_{1}(x)+\beta u_{2}(x)+\lambda u_{3}(x)
$$

is the general integral of equation (2). On substituting this value of $u$ in the expression defining $\lambda$, we obtain

so that

$$
\lambda=\int_{x_{1}}^{x_{2}}\left[p\left(a u_{1}+\beta u_{2}+\lambda u_{3}\right)+q\left(\alpha u_{1}^{\prime}+\beta u_{2}^{\prime}+\lambda u_{3}^{\prime}\right)\right] d x,
$$

$$
\lambda=\frac{\int_{x_{1}}^{x_{*}}\left[p\left(\alpha u_{1}+\beta u_{2}\right)+q\left(\alpha u_{1}^{\prime}+\beta u_{2}^{\prime}\right) d x\right.}{1-\int_{x_{1}}^{x_{*}}\left[p u_{3}+q u_{3}^{\prime}\right] d x},
$$

assuming that $1-\int_{x_{1}}^{x_{2}}\left[p u_{3}+q u_{3}^{\prime}\right] d x$ is not zero.

Hence we have proved that the general integral of equation (1) can be expressed in the form

$$
u=a u_{1}(x)+\beta u_{2}(x)+\frac{\int_{x_{1}}^{x_{2}}\left[p\left(a u_{1}+\beta u_{2}\right)+q\left(a u_{1}^{\prime}+\beta u_{2}^{\prime}\right)\right] d x}{1-\int_{x_{1}}^{x_{2}}\left[p u_{3}+q u_{3}^{\prime}\right] d x} u_{3}(x) .
$$

This solution may be written in the form

$$
u=a \bar{u}_{1}(x)+\beta \bar{u}_{2}(x),
$$


where

$$
\begin{aligned}
& \tilde{u}_{1}(x) \equiv u_{1}(x)+\frac{\int_{x_{1}}^{x_{:}}\left[p u_{1}+q u_{1}^{\prime}\right] d x}{1-\int_{x_{1}}^{x_{2}}\left[p u_{3}+q u_{3}^{\prime}\right] d x} u_{3}(x), \\
& \tilde{u}_{2}(x) \equiv u_{2}(x)+\frac{\int_{x_{1}}^{x_{2}}\left[p u_{2}+q u_{2}^{\prime}\right] d x}{1-\int_{x_{1}}^{x_{2}}\left[p u_{3}+q u_{3}^{\prime}\right] d x} u_{3}(x) .
\end{aligned}
$$

§2. It will now be shown that $\bar{u}_{1}(x)$ and $\bar{u}_{2}(x)$ are linearly independent. If this is not so, a relation $\bar{u}_{1}(x)=A \bar{u}_{2}(x)$ exists, where $A$ is a constant. This requires

$$
u_{1}+u_{3} \frac{\int_{x_{2}}^{x_{2}}\left[p u_{1}+q u_{1}^{\prime}\right] d x}{1-\int_{x_{1}}^{x_{2}}\left[p u_{3}+q u_{3}^{\prime}\right] d x}=A\left[u_{2}+u_{3} \frac{\int_{x_{2}}^{x_{3}}\left[p u_{2}+q u_{2}^{\prime}\right] d x}{1-\int_{x_{1}}^{x_{2}}\left[p u_{3}+q u_{3}^{\prime}\right] d x}\right] \text {, }
$$

from which we can deduce that either

(i) $u_{1}=A u_{2}$,

or

(ii) $u_{3}\left[\frac{\int_{x_{1}}^{x_{2}}\left[p u_{1}+q u_{1}^{\prime}\right] d x-A \int_{x_{1}}^{x_{i:}}\left[p u_{2}+q u_{2}^{\prime}\right] d x}{1-\int_{x_{1}}^{x_{*}}\left[p u_{3}+q u_{3}^{\prime}\right] d x}\right]=A u_{2}-u_{1}$.

The first result is impossible since $u_{1}$ and $u_{2}$ are linearly independent. The second states that $u_{3}(x)$ can be expressed in the form $C u_{1}(x)+D u_{2}(x)$, where $C$ and $D$ are independent of $x$; i.e. $u_{3}(x)$ is a solution of equation (3), which is impossible since it is a solution of equation (4). Thus we have proved that the general integral of equation (1) is expressible in the form

$$
u=\alpha \bar{u}_{1}(x)+\beta \bar{u}_{2}(x),
$$

where $\alpha, \beta$ are arbitrary constants, and $\bar{u}_{1}(x), \bar{u}_{2}(x)$ are linearly independent.

It will now be shown that one and only one solution of equation (1) exists which satisfies the conditions

$$
u\left(x_{0}\right)=u_{0}, \quad u^{\prime}\left(x_{0}\right)=u_{0}^{\prime},
$$

where $u_{0}$ and $u_{0}^{\prime}$ are any numbers. If $u(x)$ is a solution of equation (1) which satisfies the conditions $u\left(x_{0}\right)=u_{0}, u^{\prime}\left(x_{0}\right)=u_{0}^{\prime}$, we know that $u(x)$ is of the form

$$
\alpha u_{1}(x)+\beta u_{2}(x)+\lambda u_{3}(x),
$$


where

$$
\begin{aligned}
& u_{0}-\lambda u_{3}\left(x_{0}\right)=\alpha u_{1}\left(x_{0}\right)+\beta u_{2}\left(x_{0}\right) \\
& u_{0}^{\prime}-\lambda u_{3}^{\prime}\left(x_{0}\right)=\alpha u_{1}^{\prime}\left(x_{0}\right)+\beta u_{2}^{\prime}\left(x_{0}\right)
\end{aligned}
$$

Now, since $u_{1}$ and $u_{2}$ are linearly independent solutions of equation (3),

$$
u_{1}\left(x_{0}\right) u_{2}^{\prime}\left(x_{0}\right)-u_{2}\left(x_{0}\right) u_{1}^{\prime}\left(x_{0}\right)
$$

is not zero, and equations (7) can be solved for $a$ and $\beta$ in the form

$$
a=a+b \lambda, \quad \beta=c+d \lambda,
$$

where $a, b, c, d$ are uniquely determined. On substituting these values for $a$ and $\beta$ in equation (5), and solving for $\lambda$, we obtain

$$
\lambda=\frac{\int_{x_{1}}^{x_{2}}\left[p\left(a u_{1}+c u_{2}\right)+q\left(a u_{1}^{\prime}+c u_{2}^{\prime}\right)\right] d x}{1-\int_{x_{1}}^{x_{2}}\left[p\left(u_{3}+b u_{1}+d u_{2}\right)+q\left(u_{3}^{\prime}+b u_{1}^{\prime}+d u_{2}^{\prime}\right)\right] d x}
$$

Hence $\lambda$, and consequently $a$ and $\beta$, are uniquely determined, and we have proved that one and only one solution of equation (1) exists which satisfies the conditions $u\left(x_{0}\right)=u_{0}, u^{\prime}\left(x_{0}\right)=u_{0}^{\prime}$.

$\S 3$. From this result we can deduce at once that since $u \equiv 0$ is a solution of equation (1), it is the only one which satisfies the conditions $u\left(x_{0}\right)=0, u^{\prime}\left(x_{0}\right)=0$. In other words, a particular integral of equation ( 1 ), which is not identically zero, cannot vanish at the same time as its first derivative at any value $x_{0}$ of $x$.

We are now in a position to use the reasoning employed in discussing differential equations to show that a particular integral $u(x)$, not identically zero, has only a finite number of zeros in a given interval $(a, b)$.

If we assume that $u(x)$ has an infinite number of zeros in $(a, b)$, this set of zeros has at least one limiting point, $c$ say. Now if $u(c) \neq 0$, then, from continuity conditions, there exists a neighbourhood of $c$ in which $u(x) \neq 0$, which is impossible. Again, if $u(c)=0$, then we have just shown that $u^{\prime}(c) \neq 0$.

But

$$
u(c+h)=h\left[u^{\prime}(c)+\omega\right]
$$

where $\omega \rightarrow 0$ as $h \rightarrow 0$, so that there exists a neighbourhood of $c$ in which $c$ is the only zero of $u(x)$, which is impossible. Thus there can be only a finite number of zeros of $u(x)$ in $(a, b)$. 
If $\bar{u}_{1}(x)$ and $\bar{u}_{2}(x)$ are linearly independent solutions of equation (1), it is clear that the determinant

$$
D(x) \equiv \begin{array}{cc}
\bar{u}_{1} & \bar{u}_{2} \\
\mid \bar{u}_{1}^{\prime} & \bar{u}_{2}^{\prime}
\end{array} \mid
$$

is not identically zero. It will now be shown that $D\left(x_{0}\right) \neq 0$, for any value $x_{0}$ of $x$ in the interval under consideration. We know that one and only one solution of equation (1) exists which satisfies the conditions

so that the equations

$$
u\left(x_{0}\right)=u_{0}, u^{\prime}\left(x_{0}\right)=u_{0}^{\prime}
$$

$$
\begin{array}{ll}
u_{0}=\alpha & \bar{u}_{1}\left(x_{0}\right)+\beta \bar{u}_{2}\left(x_{0}\right), \\
u_{0}^{\prime}=\alpha \bar{u}_{1}^{\prime}\left(x_{0}\right)+\beta \bar{u}_{.2}^{\prime}\left(x_{0}\right)
\end{array}
$$

determine $a$ and $\beta$ exactly. In order that this may be possible, we must have $D\left(x_{0}\right) \neq 0$.

The usual proof of Sturm's Theorem may now be given, and thus we have the result:

If $\bar{u}_{1}(x)$ and $\bar{u}_{2}(x)$ are linearly independent solutions of equation (1), then between each two consecutive zeros of $\bar{u}_{1}$, there lies one and only one zero of $\bar{u}_{2}$.

$\$ 4$. To illustrate the method of solution and the properties of the zeros, we shall consider briefly the equation

$$
\frac{d^{2} u}{d x^{2}}+u+\sin 3 x \int_{0}^{\pi / 2}\left(u+\frac{d u}{d x}\right) d x=0
$$

The solution of this equation is

$$
u=a \cos x+\beta \sin x+\lambda / 8 \sin 3 x,
$$

where $\lambda$ is determined by the equation

$\lambda=\int_{0}^{\pi / 2}[(\alpha+\beta) \cos x+(\beta-\alpha) \sin x+\lambda / 8 \sin 3 x+3 \lambda / 8 \cos 3 x] \mathrm{d} x$, giving

$$
\lambda=24 \beta / 13 \text {. }
$$

The general solution of the equation is therefore

$$
u=\alpha \cos x+\beta[\sin x+3 / 13 \sin 3 x],
$$

where $\cos x$ and $\sin x+3 / 13$ sin $3 x$ are the linearly independent solutions, whose zeros $\left(n+\frac{1}{2}\right) \pi$ and $n \pi$ alternate. 